\title{
Spin-resolved quantum interference in graphene
}

\author{
Mark B. Lundeberg^ and Joshua A. Folk
}

\begin{abstract}
The unusual electronic properties of single-layer graphene ${ }^{1}$ make it a promising materials system for fundamental advances in physics, and an attractive platform for new device technologies. Graphene's spin-transport properties are expected to be particularly interesting, with predictions for extremely long coherence times and intrinsic spin-polarized states at zero field ${ }^{2-5}$. To test such predictions, it is necessary to measure the spin polarization of electrical currents in graphene. Here, we resolve spin transport directly from conductance features that are caused by quantum interference. These features split visibly in an in-plane magnetic field, similar to Zeeman splitting in atomic and quantum-dot systems ${ }^{6,7}$. The spin-polarized conductance features that are the subject of this work may, in the future, lead to the development of graphene devices incorporating interferencebased spin filters.
\end{abstract}

Spin currents have recently been measured in graphene using ferromagnetic contacts, an advance that shows promise for room-temperature graphene spintronics ${ }^{8-10}$. Unfortunately, many of the other tools that have been developed for studying spin-dependent phenomena in solid-state nanostructures (optical excitation, spin-resolved Coulomb blockade, and so on) have proven difficult to implement in graphene. The Zeeman-split conductance fluctuations that are the subject of this letter provide a way to detect spin-polarized currents with a straightforward electrical measurement. This is the first report, to our knowledge, of visible spin-splitting of conductance fluctuations in any material.

Flakes of graphene were deposited using well-established mechanical exfoliation techniques ${ }^{1}$ onto silicon wafers with a $\sim 300 \mathrm{~nm}$ surface oxide, then contacted with $\mathrm{Cr} / \mathrm{Au}$ leads (Fig. 1a). The two flakes used for this experiment (hereafter referred to as A and B) were selected optically and confirmed to be single layer by quantum Hall measurements (see Supplementary Information) showing the characteristic $(n+1 / 2)$ Landau quantization ${ }^{1}$. The measurements described below were carried out in a dilution refrigerator with a base temperature of $20 \mathrm{mK}$. The electron temperature was $40 \mathrm{mK}$, based on calibration using the Kondo effect in GaAs quantum dots, but the conductance fluctuations presented here were insensitive to temperature below $100 \mathrm{mK}$. The data presented here are from two-terminal conductance measurements in flake $\mathrm{A}$, with a contact spacing of $3 \mu \mathrm{m}$ (the phase coherence length was estimated to equal or exceed sample dimensions). Similar spin-resolved conductance fluctuations were observed in flake B, where the availability of multiple contacts enabled four-terminal measurements to eliminate contact effects (see Supplementary Information). A two-axis magnet provided fields up to $B_{\|}=12 \mathrm{~T}$ in the plane of the graphene and fine control of the out-of-plane component up to $B_{\perp}=120 \mathrm{mT}$ (Fig. 1a).

Carrier density $n$ was controlled capacitively with a voltage $V_{\mathrm{G}}$ on the backgate, inducing a density $n=\alpha V_{\mathrm{G}}$ in the graphene sheet; the proportionality constants of each flake were extracted from quantum Hall data. A Dirac resistance peak was observed when $V_{\mathrm{G}}$ tuned the carrier density to the charge neutrality point. The conductance, $G$, in the two-wire measurement was calculated after taking into account an estimated contact resistance of $3.2 \mathrm{k} \Omega$. Conductance fluctuations, $\delta \mathrm{G}$, were visible superimposed on the Dirac line shape (Fig. 1b), and were extracted by subtracting an ensemble-averaged background (see Supplementary Information). The mean free path ranged from 100 to $200 \mathrm{~nm}$, much smaller than sample dimensions, so transport in the flake was diffusive.

Graphene, like any metallic or semiconducting system, shows 'universal' conductance fluctuations when transport is phase coherent ${ }^{11-14}$. These aperiodic fluctuations arise from quantum interference between all possible paths that charge carriers take as they traverse the device. The interference pattern is randomized by small changes in the relative phase between trajectories, which depends on the Aharonov-Bohm magnetic flux through the sample as well as on the Fermi wave vector ${ }^{11}$. The conductance includes contributions from spin-up and spin-down carriers: $G=G_{\uparrow}+G_{\downarrow}$. In the absence of spin-orbit and many-body interactions, $G_{\uparrow / \downarrow}$ depends on the spin only through the Fermi wave vector, and therefore the density, of the respective carrier population.

Spin-up and spin-down carriers have the same density at zero field, $n_{\uparrow}=n_{\downarrow}$. In a simple picture, then, zero-field conductance fluctuations include identical contributions from both populations (Fig. 1c). Applying a magnetic field partially polarizes the graphene-it induces a difference between spin-up and spin-down carrier densities. The total density $n=n_{\uparrow}+n_{\downarrow}=\alpha V_{\mathrm{G}}$ is set by the backgate voltage; the difference in the densities $n_{\uparrow}-n_{\downarrow}=(1 / 2)(\mathrm{d} n / \mathrm{d} \epsilon) g \mu_{\mathrm{B}} B$ is set by the magnetic field, where $\mathrm{d} n / \mathrm{d} \epsilon$ is the density of states and $g \mu_{\mathrm{B}} B$ is the Zeeman energy. Together these yield

$$
n_{\uparrow / \downarrow}=\frac{\alpha}{2}\left(V_{\mathrm{G}} \pm \frac{V_{\mathrm{G}}^{\text {offset }}}{2}\right), \quad V_{\mathrm{G}}^{\text {offset }}=\frac{1}{\alpha} \frac{\mathrm{d} n}{\mathrm{~d} \epsilon} g \mu_{\mathrm{B}} B
$$

As a result, spin-up and spin-down conductance contributions at finite field are offset in gate voltage by $V_{\mathrm{G}}^{\text {offset }}$, leading to Zeeman splitting of interference features in a gate-voltage trace $\mathrm{G}\left(\mathrm{V}_{\mathrm{G}}\right)$ (Fig. 1d).

Zeeman splitting of graphene conductance fluctuations can be seen in Fig. 1e,f. The ' $\mathrm{V}$ ' shapes in the data correspond to spin-resolved conductance: left-moving (right-moving) arms reflect interference for a particular density of spin-down (spin-up) carriers. Although conductance fluctuations have been observed for several decades in a wide variety of materials ${ }^{11,13}$, a direct measurement of their Zeeman splitting has never before been reported. Several factors must conspire to make this effect visible. A low density of states enhances the visibility of conductance fluctuations and reduces the gate-voltage offset between spinsplit features; a small spin-orbit interaction is required for the approximation of two independent spin populations to be valid; atomic-scale flatness and precise alignment of the in-plane field reduce the effective Aharonov-Bohm flux entrained by an in-plane 
a

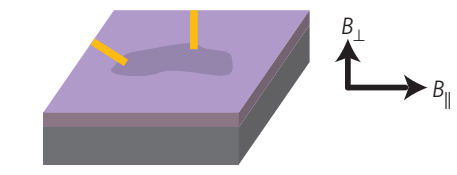

C
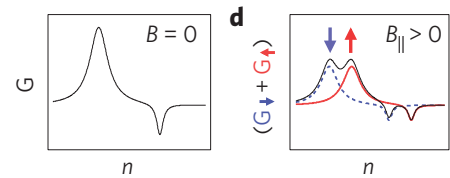

e

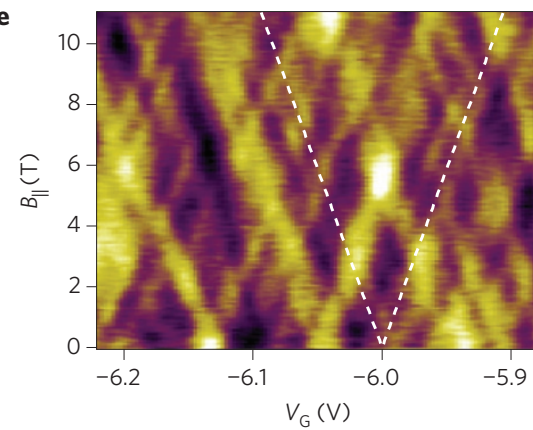

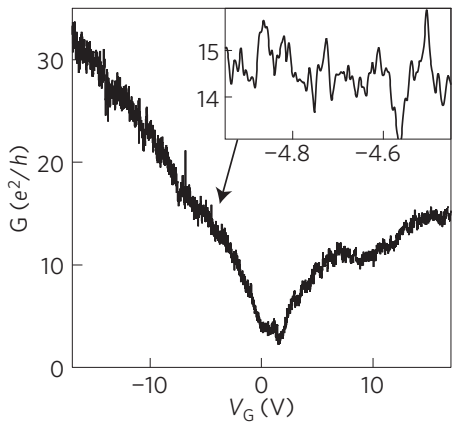

f

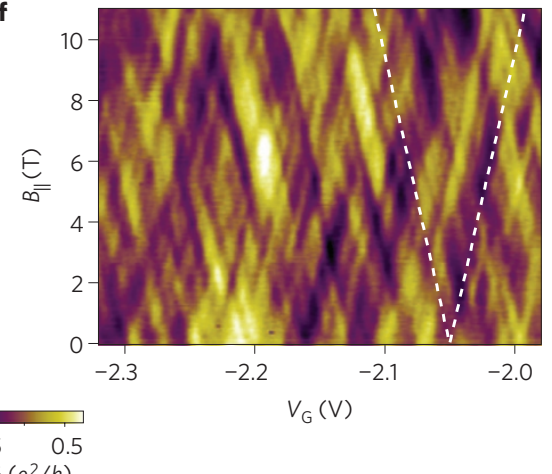

$\delta \mathrm{G}\left(e^{2} / h\right)$

Figure 1 | Zeeman effect in graphene. a, Schematic of a single-layer graphene device, showing the orientation of the magnetic fields $B_{\|}$and $B_{\perp}$.

b. Two-terminal conductance, $\mathrm{G}$, measured as a function of backgate voltage $V_{\mathrm{G}}$ at $20 \mathrm{mK}$, showing the Dirac conductance minimum ( $B_{\perp}=B_{\|}=20 \mathrm{mT}$ ). Inset: A sample of conductance fluctuations over a narrow range of gate voltage. $\mathbf{c}, \mathbf{d}$, Diagrams of the spin-splitting effect: a simulated conductance trace

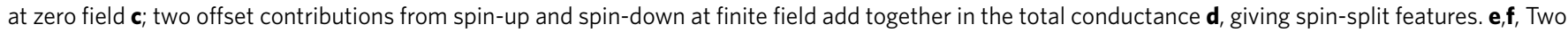
examples of conductance fluctuations spin-splitting in an in-plane field, taken over two ranges of gate voltage ( $\left.B_{\perp}=80 \mathrm{mT}\right)$. For both images, a smooth background was subtracted to highlight fluctuations. The offset (indicated by the dashed lines, as guides to the eye) owing to spin is larger around $-6 \mathrm{~V}$ compared with $-2 \mathrm{~V}$ because the density of states increases with density. The dashed lines correspond to densities of states of (e) $12 \times 10^{12}$ and (f) $7 \times 10^{12} \mathrm{~cm}^{-2} \mathrm{eV}^{-1}$.

magnetic field. The first three factors are naturally present in graphene; the fourth was facilitated by a two-axis magnet.

A statistical analysis of the spin-split offset at particular values of $B_{\|}$was obtained using an ensemble of traces, $\delta \mathrm{G}\left(\mathrm{V}_{\mathrm{G}}\right)$, collected for a range of out-of-plane fields, $3 \mathrm{mT} \leq B_{\perp} \leq 120 \mathrm{mT}\left(\ll B_{\|}\right)$. The offset is barely visible in the raw conductance data (Fig. 2a), but can be seen more clearly by computing an autocorrelation $C_{[\delta \mathrm{G}]}\left(\Delta V_{\mathrm{G}}\right)=\left\langle\delta \mathrm{G}\left(V_{\mathrm{G}}\right) \delta \mathrm{G}\left(V_{\mathrm{G}}+\Delta V_{\mathrm{G}}\right)\right\rangle$. Side peaks in the autocorrelation at $\Delta V_{\mathrm{G}}= \pm V_{\mathrm{G}}^{\text {offset }}$ (Fig. 2b) reflect similar features offset in the conductance traces (Fig. 2a) owing to the difference in spin-up and spin-down carrier densities.

The side peaks shifted outward with increasing $B_{\|}$(Fig. 3a), at the same time as the height of the central peak $\left(C_{[\delta \mathrm{G}]}(0)\right.$, the variance of the fluctuations) dropped by a factor of two (Fig. 3b). The drop in $C_{[\delta G]}(0)$ reflects a suppression of conductance fluctuations when the Zeeman energy, $g \mu_{\mathrm{B}} B$, exceeds energy broadening owing to temperature or dephasing. This effect has been used to characterize spin degeneracy in other systems ${ }^{15-17}$, where splitting could not be observed directly. The suppression factor of $\sim 2$ indicates that spin degeneracy was intact at zero field, after taking into account broken time-reversal symmetry owing to the small out-of-plane field. The shift of the side-peak location, $V_{\mathrm{G}}^{\text {offset }}$, with $B_{\|}$was expected from equation (1) — a statistical confirmation of the linear spin-splitting seen in Fig. 1e,f.

The density of states can be extracted directly from $V_{\mathrm{G}}^{\text {offset }}$ at a given field using $g=2$ for graphene (see Supplementary Information) and the value $\alpha=8.05 \pm 0.05 \times 10^{10} \mathrm{~cm}^{-2} \mathrm{~V}^{-1}$ determined from quantum Hall measurements (equation (1)). $V_{\mathrm{G}}^{\text {offset }}\left(V_{\mathrm{G}}\right)$ was recorded over the full gate-voltage range by computing the autocorrelation $C_{[\delta G]}\left(\Delta V_{\mathrm{G}}\right)$ within a sliding window
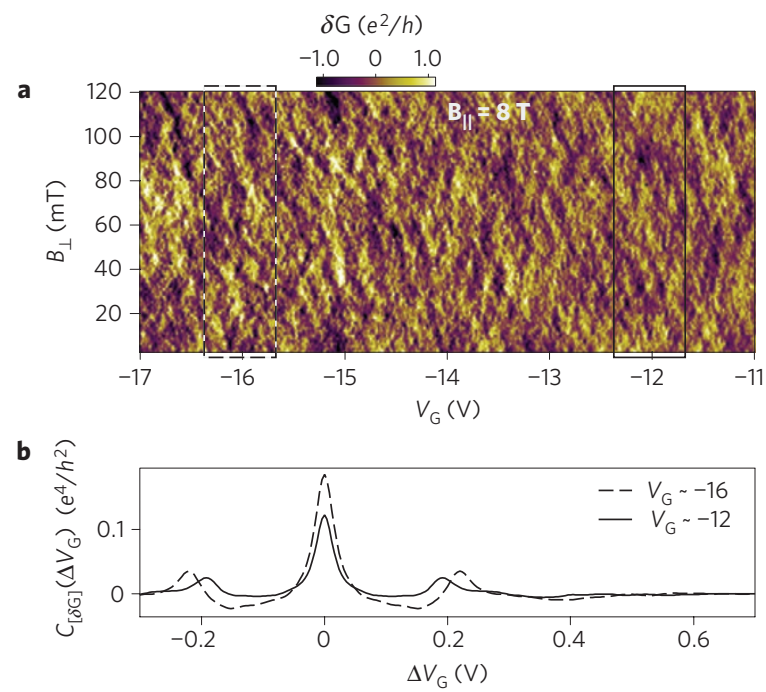

Figure 2 | Autocorrelation extraction of spin-splitting. a, Features are doubled in an image of conductance fluctuations at $B_{\|}=8 \mathrm{~T}$.

b. Autocorrelations of the windows indicated in a show that the spin-split offset (the location of the autocorrelation side peak) changes from $V_{G}^{\text {offset }}=0.22 \mathrm{~V}$ at $V_{G}=-16 \mathrm{~V}$, to $V_{G}^{\text {offset }}=0.19 \mathrm{~V}$ at $V_{G}=-12 \mathrm{~V}$. The autocorrelation function is symmetric, so side peaks appear at $\pm V_{G}^{\text {offset }}$.

in $V_{\mathrm{G}}$ (Fig. 3c). The resulting square-root line shape can be compared to $\mathrm{d} n / \mathrm{d} \epsilon$ expected from graphene's dispersion relation: $(\mathrm{d} n / \mathrm{d} \epsilon)\left(V_{\mathrm{G}}\right)=\left(2 \sqrt{\alpha / \pi} / \hbar v_{\mathrm{F}}\right) \sqrt{\left|V_{\mathrm{G}}-V_{0}\right|}$, with a Fermi velocity 


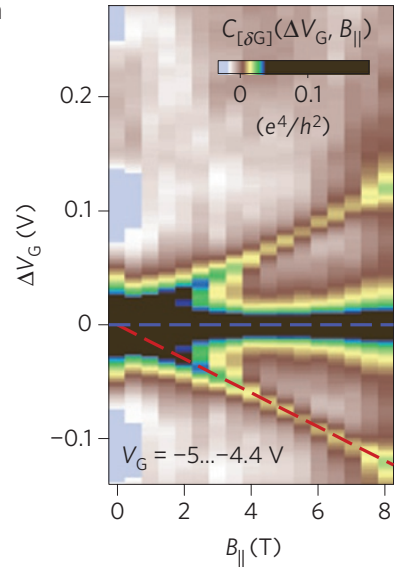

b

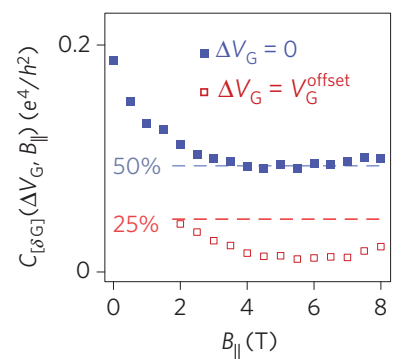

c

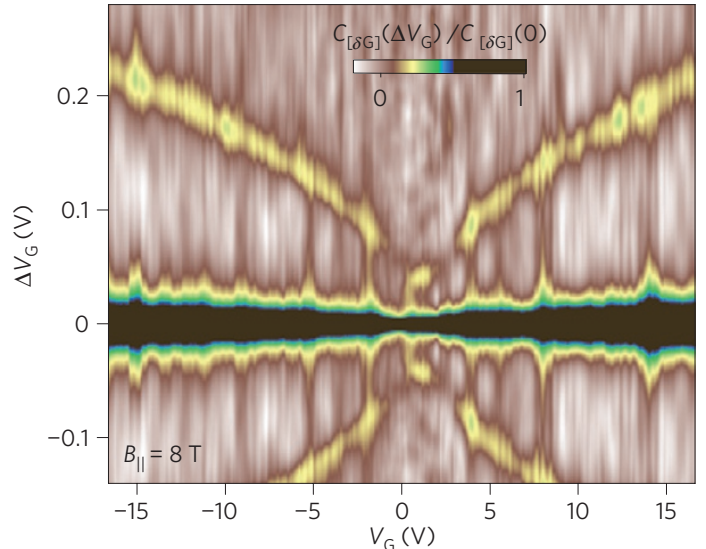

d

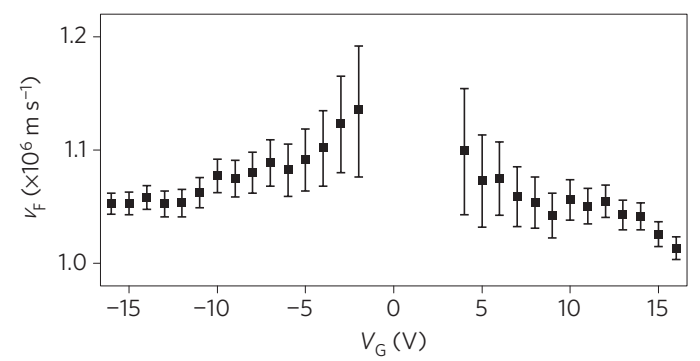

Figure 3 | Magnetic-field- and gate-dependence of spin splitting. a, Autocorrelations, $C_{[\delta G]}\left(\Delta V_{G}, B_{\|}\right)$, of fluctuations in the range $V_{G}=-5 \ldots-4.4 V$, averaged over an ensemble of traces for $3 \mathrm{mT} \leq B_{\perp} \leq 120 \mathrm{mT}$, demonstrate the in-plane field dependence of side-peak location, $V_{G}^{\text {offset }}$. The dashed red line is fitted to $V_{G}^{\text {offset }}$, and corresponds to a density of states $1.04 \times 10^{13} \mathrm{~cm}^{-2} \mathrm{eV}^{-1}$. $\mathbf{b}$, Variance $C_{[\delta G]}\left(\Delta V_{\mathrm{G}}=0, B_{\|}\right)$(filled squares) and autocorrelation side-peak height $C_{[\delta G]}\left(\Delta V_{G}=V_{G}^{\text {offset }}, B_{\|}\right)$(open squares) from a. The variance drops to the expected $50 \%$ of its zero-field value, but the side peaks fall below the expected $25 \%$. The out-of-plane field $B_{\perp} \geq 3 \mathrm{mT}$ was large enough to break time-reversal symmetry even at $B_{\|}=0$. c, Autocorrelations computed for a sliding $0.75-V$-wide window in $V_{G}$ (see Fig. 2), and averaged over an ensemble of traces for $3 \mathrm{mT} \leq B_{\perp} \leq 120 \mathrm{mT}$, show how the density of states depends on density. $\mathbf{d}$. The Fermi velocity $v_{F}$ extracted from side-peak locations, $V_{G}^{\text {offset }}$, in $\mathbf{c}$ is enhanced near the Dirac point. The error bars are primarily due to uncertainty in the charge neutrality point, $V_{0}=1.0 \pm 0.3 \mathrm{~V}$.

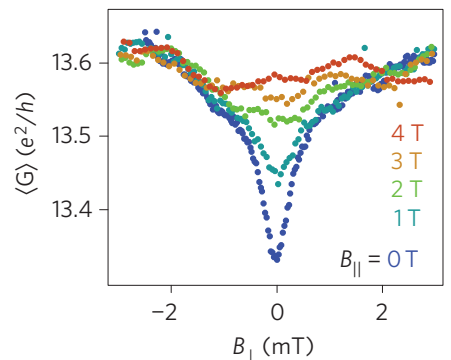

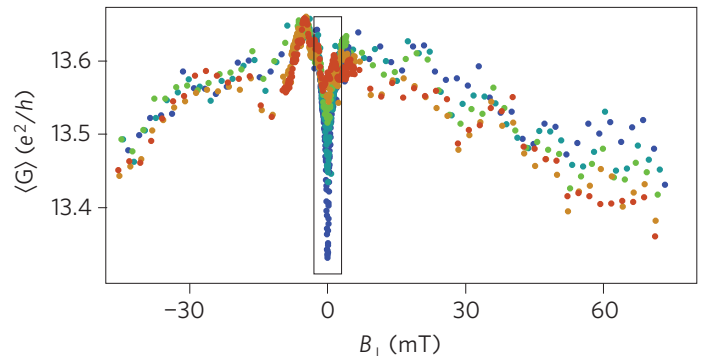

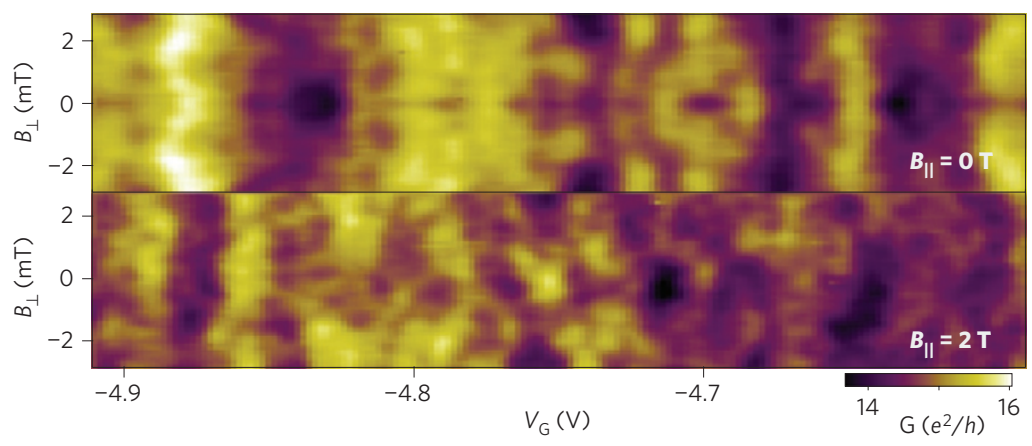

Figure 4 | Loss of weak localization and symmetry due to in-plane field. a, Averaging out the conductance fluctuations over a range $V_{G}=-5 \ldots-3 V_{\text {, }}$ weak localization dip in conductance is present at $\mathbf{B}=0$ but disappears for increasing $B_{\|}$or $B_{\perp}$. This indicates a breaking of time-reversal symmetry from either component, but at very different field scales. Curves at different $B_{\|}$are vertically offset to align $\mathrm{G}\left(B_{\perp}>10 \mathrm{mT}\right.$ ) (see Supplementary Information). b, Negative magnetoconductance for $B_{\perp}>10 \mathrm{mT}$ is unaffected by $B_{\|}$. The rectangle encloses the range from a. $\mathbf{c}, \mathbf{d}$, Conductance is symmetric in $B_{\perp}$ at $B_{\|}=0$ (c) but not at $B_{\|}=2 \mathrm{~T}(\mathbf{d})$. These data provide further evidence of time-reversal symmetry breaking by the in-plane field, excluding (for example) that the disappearance of weak localization in $\mathbf{a}$ and $\mathbf{b}$ is due to decoherence. 
$v_{\mathrm{F}}$ that would be independent of density for an ideal Dirac band structure.

As $V_{\mathrm{G}}^{\text {offset }}$ can be determined very accurately from $C_{[\delta \mathrm{G}]}\left(\Delta V_{\mathrm{G}}\right)$, spin-split fluctuations provide an accurate measure of $v_{\mathrm{F}}$. Experimental error bars were typically one or two per cent of the measured value (Fig. 3d), dominated by uncertainty in the charge neutrality point, $V_{0}$. Both flakes showed $v_{\mathrm{F}}>9 \times 10^{5} \mathrm{~m} \mathrm{~s}^{-1}$ away from the Dirac peak, with $v_{\mathrm{F}}>1.05 \times 10^{6}$ throughout most of the gate-voltage range for flake A (Fig. 3d) and for the electron-doped gate-voltage range of flake B (see Supplementary Information). These values are similar to those reported elsewhere ${ }^{18-20}$ but considerably larger than the expected $v_{\mathrm{F}} \approx 0.8 \times 10^{6} \mathrm{~m} \mathrm{~s}^{-1}$ from non-interacting band-structure calculations ${ }^{4}$. Enhancements in $v_{\mathrm{F}}$ have been predicted due to many-body effects ${ }^{21-23}$. On the other hand, differences in the Fermi speeds for flakes A and B suggest that the apparent Fermi speed in realistic graphene samples also depends strongly on disorder and other sample details. Larger values of $v_{\mathrm{F}}$ are observed in Fig. 3d at lower densities, similar to a report of infrared spectroscopy measurements ${ }^{19}$, but in flake $\mathrm{B}$ the trend was reversed, providing further evidence for sample-to-sample variations in Fermi speed.

To observe V shapes such as those shown in Fig. 1e,f, interference features must shift, but not otherwise change, as a function of in-plane field. In other words, the primary influence of the magnetic field on the interference must be through its effect on the densities of spin-up and spin-down carriers, rather than a change in Aharonov-Bohm flux. Fields of a tesla or more were required to differentiate the two carrier densities, but the Aharonov-Bohm flux introduced by a few millitesla in $B_{\perp}$ was sufficient to alter the observed interference pattern. The drastically different field scales for spin and Aharonov-Bohm effects demanded precise alignment (within $0.05^{\circ}$ ) of the $B_{\|}$axis to the plane of the graphene flake. The alignment of $B_{\|}$was monitored using weak localization, the coherent enhancement of backscattering associated with time-reversal symmetry at $B_{\perp}=0$ (ref. 11). Slight mis-alignment led to a shift in the location of the weak-localization conductance dip as $B_{\|}$was raised, and was corrected by offsetting $B_{\perp}$.

In addition to shifting, the weak-localization dip also decreased in magnitude with $B_{\|}$, by a factor of two at $1 \mathrm{~T}$ and below detectable levels above $4 \mathrm{~T}$ (Fig. 4a,b). The complete collapse of the weak-localization dip, over a field range where the variance of conductance fluctuations decreases only by a factor of two, implies that time-reversal symmetry is broken even by an in-plane field ${ }^{24,25}$. The disappearance of symmetry in $g\left(B_{\perp}\right) \leftrightarrow g\left(-B_{\perp}\right)$ at finite $B_{\|}$(Fig. $\left.4 \mathrm{c}, \mathrm{d}\right)$ provided further evidence of time-reversal symmetry breaking ${ }^{24}$. Similar phenomena have been observed in semiconductor two-dimensional electron gases, and have been associated with finite thickness and nanometre-scale undulations in the two-dimensional electron gas ${ }^{25}$, allowing $B_{\|}$to thread an Aharonov-Bohm flux through the conductor. The $B_{\|}$-scale of weak-localization collapse in graphene corresponds to an effective thickness of $\sim 1 \mathrm{~nm}$, in agreement with previous measurements of the intrinsic ripple size in graphene sheets ${ }^{26,27}$.

The ability to distinguish conductance fluctuations associated with spin-up transport from those associated with spin-down transport, using the magnetic-field dependence of their position in gate voltage, may allow the development of interference-based spin filters in graphene. The maximum spin polarization of current injected through such a device is set by the ratio of conductance fluctuation amplitude to the conductance itself. Although this ratio was typically $<10 \%$ in the devices measured here, it could be increased by using constrictions or other device geometries to decrease the overall conductance, because the amplitude of conductance fluctuations remains large as long as the device size does not exceed the phase coherence length.
Received 15 April 2009; accepted 1 September 2009; published online 11 October 2009

\section{References}

1. Novoselov, K. S. et al. Electric field effect in atomically thin carbon films. Science 306, 666-669 (2004).

2. Yazyev, O. V. Hyperfine interactions in graphene and related carbon nanostructures. Nano Lett. 8, 1011-1015 (2008).

3. Kane, C. L. \& Mele, E. J. Quantum spin hall effect in graphene. Phys. Rev. Lett 95, 226801 (2005).

4. Peres, N. M. R., Guinea, F. \& Castro Neto, A. H. Coulomb interactions and ferromagnetism in pure and doped graphene. Phys. Rev. B 72, 174406 (2005).

5. Wimmer, M., Adagideli, İ., Berber, S., Tománek, D. \& Richter, K. Spin currents in rough graphene nanoribbons: Universal fluctuations and spin injection. Phys. Rev. Lett. 100, 177207 (2008).

6. Zeeman, P. The effect of magnetisation on the nature of light emitted by a substance. Nature 55, 347 (1897).

7. Cobden, D. H., Bockrath, M., McEuen, P. L., Rinzler, A. G. \& Smalley, R. E. Spin splitting and even-odd effects in carbon nanotubes. Phys. Rev. Lett. 81, 681-684 (1998).

8. Tombros, N., Jozsa, C., Popinciuc, M., Jonkman, H. T. \& van Wees, B. J. Electronic spin transport and spin precession in single graphene layers at room temperature. Nature 448, 571-574 (2007).

9. Cho, S., Chen, Y.-F. \& Fuhrer, M. S. Gate-tunable graphene spin valve. Appl. Phys. Lett. 91, 123105 (2007)

10. Ohishi, M. et al. Spin injection into a graphene thin film at room temperature. Jpn. J. Appl. Phys. 46, L605-L607 (2007).

11. Beenakker, C. W. J. \& van Houten, H. Quantum transport in semiconductor nanostructures. Solid State Phys. 44, 1-228 (1991).

12. Kharitonov, M. Y. \& Efetov, K. B. Universal conductance fluctuations in graphene. Phys. Rev. B 78, 033404 (2008).

13. Morozov, S. V. et al. Strong suppression of weak localization in graphene. Phys. Rev. Lett. 97, 016801 (2006).

14. Horsell, D. et al. Mesoscopic conductance fluctuations in graphene. Solid State Commun. 149, 1041-1045 (2009).

15. Moon, J. S., Birge, N. O. \& Golding, B. Observation of universal conductance-fluctuation crossovers in mesoscopic Li wires. Phys. Rev. B 56, 15124-15132 (1997)

16. Debray, P., Pichard, J.-L., Vicente, J. \& Tung, P. N. Reduction of mesoscopic conductance fluctuations due to Zeeman splitting in a disordered conductor without spin-orbit scattering. Phys. Rev. Lett. 63, 2264-2267 (1989).

17. Folk, J. A. et al. Spin degeneracy and conductance fluctuations in open quantum dots. Phys. Rev. Lett. 86, 2102-2105 (2001).

18. Deacon, R. S., Chuang, K.-C., Nicholas, R. J., Novoselov, K. S. \& Geim, A. K. Cyclotron resonance study of the electron and hole velocity in graphene monolayers. Phys. Rev. B 76, 081406 (2007).

19. Li, Z. et al. Dirac charge dynamics in graphene by infrared spectroscopy. Nature Phys. 4, 532-535 (2008).

20. Martin, J. et al. Observation of electron-hole puddles in graphene using a scanning single-electron transistor. Nature Phys. 4, 144-148 (2008).

21. Sarma, S. D., Hwang, E. H. \& Tse, W.-K. Many-body interaction effects in doped and undoped graphene: Fermi liquid versus non-Fermi liquid. Phys. Rev. B 75, 121406 (2007)

22. Trevisanutto, P. E., Giorgetti, C., Reining, L., Ladisa, M. \& Olevano, V. Ab initio GW many-body effects in graphene. Phys. Rev. Lett. 101, 226405 (2008).

23. Polini, M., Asgari, R., Barlas, Y., Pereg-Barnea, T. \& MacDonald, A. Graphene: A pseudochiral Fermi liquid. Solid State Commun. 143, 58-62 (2007).

24. Zumbühl, D. M. et al. Orbital effects of in-plane magnetic fields probed by mesoscopic conductance fluctuations. Phys. Rev. B 69, 121305 (2004).

25. Mensz, P. M. \& Wheeler, R. G. Magnetoconductance due to parallel magnetic fields in silicon inversion layers. Phys. Rev. B 35, 2844-2853 (1987).

26. Meyer, J. C. et al. The structure of suspended graphene sheets. Nature 446, 60-63 (2007).

27. Ishigami, M., Chen, J. H., Cullen, W. G., Fuhrer, M. S. \& Williams, E. D. Atomic structure of graphene on $\mathrm{SiO}_{2}$. Nano Lett. 7, 1643-1648 (2007).

\section{Acknowledgements}

We acknowledge V. F'alko, D. Goldhaber-Gordon, H. Heersche, T. Ihn, G. Kamps, C.-Y. Lo, A. Morpurgo and K. Todd for helpful discussions. Graphite was provided by S. Fain and D. Cobden. M.B.L. acknowledges a PGS-D from NSERC; work financially supported by CIFAR, CFI and NSERC.

\section{Author contributions}

Sample fabrication, measurement, analysis and write up by M.B.L., under the supervision of J.A.F

\section{Additional information}

Supplementary information accompanies this paper on www.nature.com/naturephysics. Reprints and permissions information is available online at http://npg.nature.com/ reprintsandpermissions. Correspondence and requests for materials should be addressed to M.B.L. 